\title{
Evaluation of a portable assembly catheter simulator using a 3D-printed heart model for percutaneous transvenous mitral commissurotomy in developing countries
}

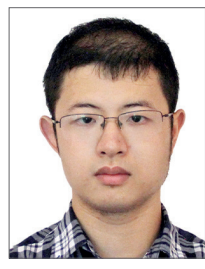

Xiaoyang Song ${ }^{1}, \mathrm{MD}$; Naritatsu Saito ${ }^{*}, \mathrm{MD}$; Yoshimasa Nagata ${ }^{2}$; Afzalur Rahman $\mathrm{MD}^{3}$; Martin M. Murage 4 , MD; Farhana Ahmed ${ }^{3}$, MD; Mohammad Arifur Rahman ${ }^{3}$, MD; Tariq Ahmed Chowdhury ${ }^{3}$, MD; Bernard Muriuki Gitura ${ }^{4}, \mathrm{MD}$; Christine Awuor Yuko-Jowi, MD; Peter Muriithi Nyamu, MD; Takeshi Kimura ${ }^{4}, \mathrm{MD}$; Kanji Inoue ${ }^{2}, \mathrm{MD}$

1. Department of Cardiovascular Medicine, Graduate School of Medicine, Kyoto University, Kyoto, Japan; 2. PTMC institute, Kyoto, Japan; 3. National Institute of Cardiovascular Disease, Dhaka, Bangladesh; 4. Kenyatta National Hospital, Nairobi, Kenya

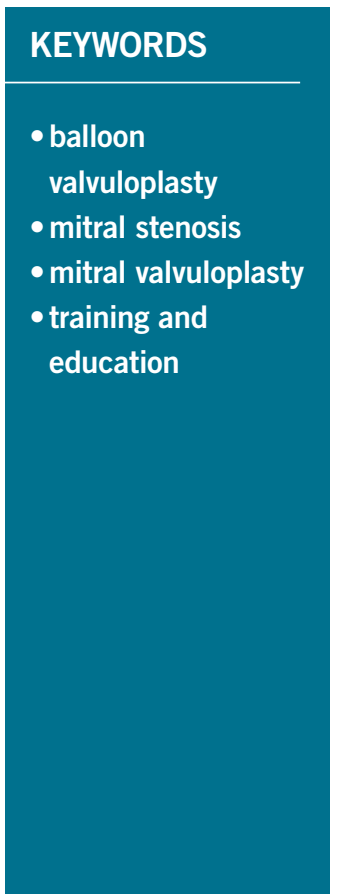

\begin{abstract}
Aims: We developed a catheter simulator for percutaneous transvenous mitral commissurotomy (PTMC) based on the data from a patient with mitral valve stenosis. The simulator has the following characteristics: 1) the simulator is portable and easy to assemble and disassemble, 2) the cardiac portion is created using a 3D-printer, based on patient computed tomography data, 3) the simulator uses a foot-operated water pump to create pulsatile flow, and 4) the fossa ovalis in the atrial septum of the heart model is made of a thin polyurethane membrane and is interchangeable. We aimed to assess the effectiveness of this novel simulator for training in PTMC using the Inoue balloon in developing countries.
\end{abstract}

Methods and results: We used this simulator for training in the National Institute of Cardiovascular Diseases in Bangladesh (13 physicians), and in Kenyatta National Hospital in Kenya (11 physicians). The effectiveness of training was evaluated by questionnaire and the procedure time in simulation. The questionnaire obtained from the trainees showed that the model scored $4.7 \pm 0.5$ for realism, utility of pulsatile flow scored $4.7 \pm 0.5$, simulator utility scored $4.9 \pm 0.3$, and the effect of training on PTMC performance scored $4.9 \pm 0.5$. The procedure time in simulation was shortened from $30.0 \pm 12.6 \mathrm{~min}$ (first time), to $23.4 \pm 11.9 \mathrm{~min}$ (second time) and to $20.4 \pm 11.1 \mathrm{~min}$ (third time) $(\mathrm{p}<0.01)$.

Conclusions: The novel portable assembly catheter simulator using a 3D-printed heart model for PTMC received positive comments and improved the skills of trainees.

\footnotetext{
*Corresponding author: Department of Cardiovascular Medicine, Kyoto University Graduate School of Medicine, 54 Kawahara-cho, Shogoin, Sakyo-ku, Kyoto, 606-8507, Japan.E-mail:naritatu@kuhp.kyoto-u.ac.jp
} 


\section{Abbreviations}

PTMC percutaneous transvenous mitral commissurotomy

CT computed tomography

RHD rheumatic heart disease

NICVD National Institute of Cardiovascular Diseases

KNH Kenyatta National Hospital

JICA Japan International Cooperation Agency

\section{Introduction}

Rheumatic fever is a leading cause of valvular heart disease, especially in developing countries with large populations and poor quality public health provisions, and rheumatic heart disease (RHD) is a major public health problem ${ }^{1,2}$. Therefore, there is a need for skilled cardiologists, and especially interventionists, to treat rheumatic valvular disease. However, there is often a shortage of skilled physicians, and the training of qualified medical teams is hindered by socioeconomic factors ${ }^{3}$. In light of these factors, teams from other countries have directly assisted local surgeons, and the effort has achieved considerable suc$\operatorname{cess}^{3,4}$. However, the number of patients who benefit from this approach is limited. Training a local heart team is a reasonable solution. Teaching catheter intervention techniques is a challenge, and simulation-based training is reportedly effective. However, quantitative assessment of simulation-based training has not been reported. We developed a novel catheter simulator for the training of percutaneous transvenous mitral commissurotomy (PTMC). The purpose of the present study was to assess the effectiveness of this novel simulator for training PTMC using the Inoue balloon in developing countries.

\section{Methods}

The simulator consisted of a water tank (length $500 \mathrm{~mm}$, width $385 \mathrm{~mm}$, depth $170 \mathrm{~mm}$ ), a heart model, a rubber tube representing the inferior vena cava and femoral vein, and a foot-operated water pump (Figure 1). The heart model was constructed with a 3D printer (crossEffect Inc., Kyoto, Japan) using polyurethane resin, based on patient computed tomography (CT) data. The cardiac portion of the simulator was translucent, and the right and left ventricles were omitted to better visualise the catheter movement. The fossa ovalis in the atrial septum of the heart model was made of a thin polyurethane membrane and was interchangeable. The simulator used a foot pump to create physiological pulsatile flow. The pump drew water from the water tank and discharged water into the left atrium. The suction tube was connected to a translucent cup. By covering the mitral annulus with the translucent cap, a pulsatile flow that sucked the Inoue balloon catheter into the translucent cap was made (Figure 2). Utilising the flow to navigate the Inoue balloon catheter into the left ventricle is an important technique in PTMC. Physicians can practice this technique with this simulator.

The effectiveness of the simulation was evaluated by conducting questionnaire surveys after the simulation, and measuring the change in procedure time. In the questionnaire, the participants rated the realism and training potential of the simulator on a Likert scale from 1 (poor) to 5 (excellent) (Figure 3). The procedure time (min) was defined as the time from insertion of the $7 \mathrm{Fr}$ sheath to passage of the Inoue balloon into the left ventricle. We used the procedure time as an objective index to evaluate trainee improvement. This training programme aimed at transferring knowledge and developing skills in local heart teams.

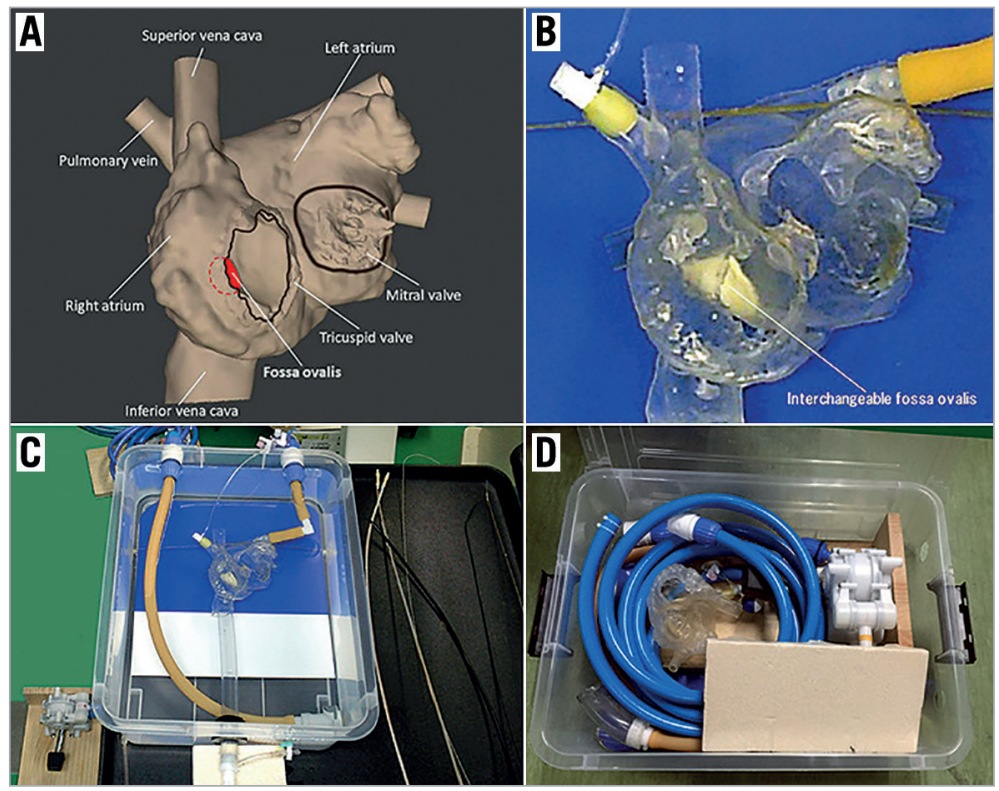

Figure 1. Percutaneous transvenous mitral commissurotomy simulator using a 3D-printed heart model. A) Design of the heart model (frontal view). The right and left ventricles are omitted to better visualise the catheter movement. B) The cardiac portion is created using a 3D-printer based on patient computed tomography data. The fossa ovalis in the atrial septum is made of an interchangeable thin polyurethane membrane. C) Overall view of the simulator. D) Simulator packed away in its water tank. 


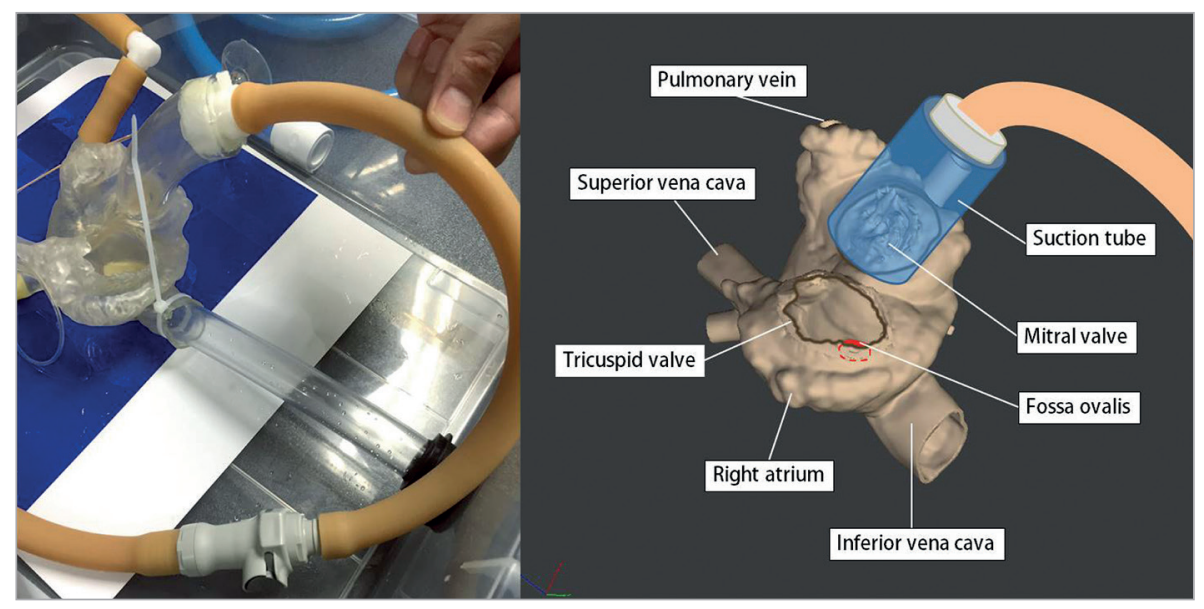

Figure 2. The translucent cap of the suction tube covering the mitral annulus. The suction tube is connected to a foot pump creating pulsatile flow that sucks the Inoue balloon catheter into the translucent cap.

\section{Statistics}

Statistical analyses were performed using JMP 14 Pro (SAS Institute Inc, Cary, NC, USA) software. Continuous variables were presented as mean \pm standard deviation. A 2-sided p-value of $<0.05$ was considered statistically significant.

Evaluation of PTMC simulator using foot pump for producing pulsatile flow

घq, 20XX

\section{Questionnaire}

\begin{tabular}{|c|c|c|c|c|c|}
\hline Name: & & & $\mathrm{Ag}$ & years & \\
\hline How many y & work & al do & & & years \\
\hline How many y & work & ventic & jist? & & years \\
\hline Have you ev & PTN & & & Yes / No & \\
\hline Have you ex & AC us & ballo & & Yes / No & \\
\hline Have you ev & the & sed tr & & Yes / No & \\
\hline Realism of & & & & & \\
\hline 1 & 2 & 3 & 4 & & 5 \\
\hline Not Realistic & & & & & Realistic \\
\hline Do you thin & e flor & & latio & & \\
\hline 1 & 2 & 3 & 4 & & 5 \\
\hline Disagree & & & & & Agree \\
\hline
\end{tabular}

This model is useful to practice the real case on the simulator, prior to performing the real PTMC procedure.

\begin{tabular}{|c|c|c|c|c|}
\hline & & & & \\
\hline 1 & 2 & 3 & 4 & 5 \\
\hline Disagree & & & & Agree \\
\hline \multicolumn{5}{|c|}{ Does this simulation-based training change your PTMC practice pattern? } \\
\hline 1 & 2 & 3 & 4 & 5 \\
\hline No & & & & Yes \\
\hline
\end{tabular}

Simulation Time Procedure time (15t): Procedure time $\left(2^{\text {nd }}\right)$ : Procedure time (3ro):

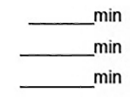

Figure 3. Questionnaire for assessing efficacy of catheter simulator training.

\section{Results}

The simulator-based training was conducted in the National Institute of Cardiovascular Diseases (NICVD), Bangladesh (13 physicians in 5 days from November 4-8, 2018). The training was also conducted in Kenyatta National Hospital (KNH), Kenya (11 physicians in 5 days from December 1-5, 2019). The average age of training participants was $45.1 \pm 6.7$ years old (NICVD: $45.1 \pm 6.7$, KNH: $49.3 \pm 6.9$ ), the average years of experience as physicians was $20.1 \pm 7.0$ (NICVD: $17.1 \pm 4.3$, KNH: 23.6 \pm 8.1 ), and the average years of experience as interventional cardiologists was $7.1 \pm 5.2$ (NICVD: $5.8 \pm 2.9$, KNH: $8.7 \pm 6.8$ ). 22 out of 24 physicians (91.7\%) had experience in PTMC mainly as assistants (NICVD: 13/13 [100\%], KNH: 9/11 [81.8\%]), and 8 (62\%) had experience in PTMC using the Inoue balloon (NICVD: 8/13 [61.5\%], KNH: 3/11 [27.2\%]). Only 4 out of 24 physicians (16.7\%) had experienced simulation-based training for other catheter intervention procedures (NICVD: 2/13 [15.4\%], KNH: $2 / 11[18.1 \%])$.

The questionnaires completed by all the trainees after the course showed that: realism of the model scored $4.7 \pm 0.5$ (NICVD: $4.8 \pm 0.4$, KNH: $4.3 \pm 0.8$ ), utility of pulsatile flow scored $4.7 \pm 0.6$ (NICVD: 5 [all participants scored 5], KNH: 4.5 \pm 0.5 ), simulator utility scored $4.9 \pm 0.3$ (NICVD: $4.8 \pm 0.4, \mathrm{KNH}$ : $4.9 \pm 0.3$ ), and the effect of training on PTMC performance scored $4.9 \pm 0.4$ (NICVD: $4.9 \pm 0.3, \mathrm{KNH}: 4.8 \pm 0.6$ ). The procedure time in the simulation was shortened from $30.0 \pm 12.6 \mathrm{~min}$ on the first attempt (NICVD: $31.5 \pm 10.9$, KNH: $27.5 \pm 15.6$ ), to $23.4 \pm 11.9 \mathrm{~min}$ on the second attempt (NICVD: $26.1 \pm 10.4$, KNH: $20.1 \pm 13.6$ ), and $20.4 \pm 11.1 \mathrm{~min}$ on the third attempt (NICVD: $20.8 \pm 10.8, \mathrm{KNH}$ : $19.9 \pm 12.3$ ) (Figure 4, $<<0.01$, paired t-test). The procedure time in simulation was not recorded for three trainees in $\mathrm{KNH}$.

\section{Discussion}

The main findings of the present study were that: 1) a novel portable assembly catheter simulator using a 3D-printed heart model 


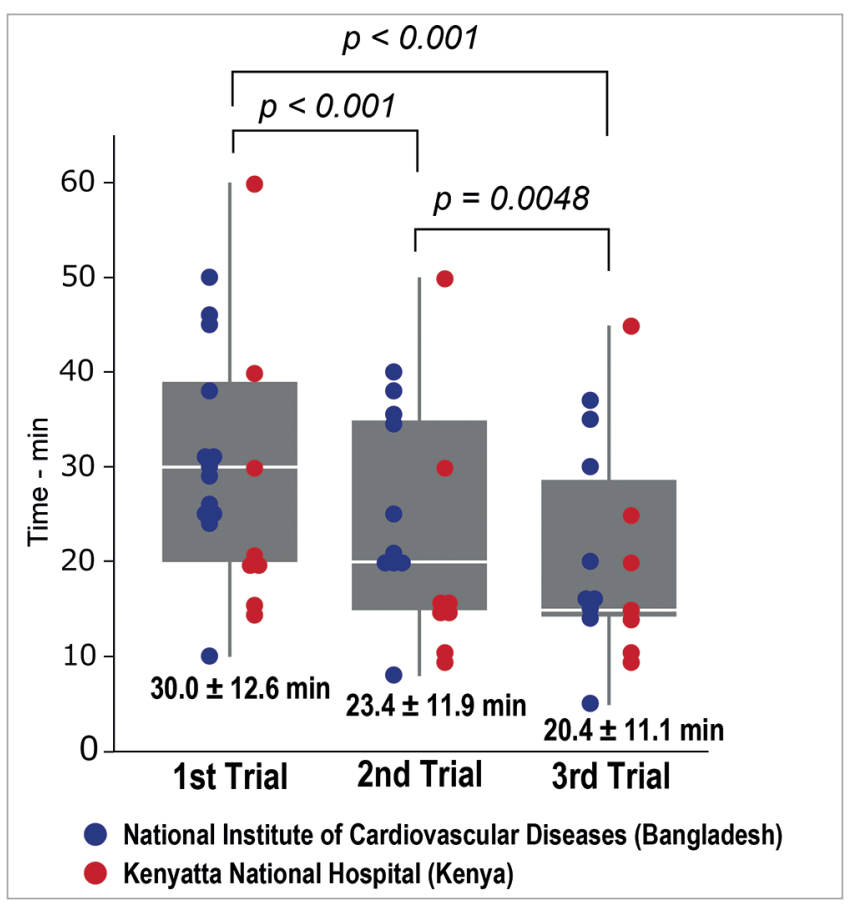

Figure 4. Change in simulation procedure time during training. The blue marker indicates the procedure time of each participant in National Institute of Cardiovascular Disease in Bangladesh, and the red marker indicates the procedure time of each participant in Kenyatta National Hospital in Kenya. Note that the procedure time in simulation was not recorded in three trainees in $\mathrm{KNH}$.

with a foot pump to create pulsatile flow for PTMC was effective for training; 2) the simulator was highly rated by the participants as close to actual treatment, and 3) the training shortened the procedure time in simulation.
Mitral valve stenosis is a common valvular sequela of acute rheumatic fever. There are nearly 33 million people with RHD globally, contributing to approximately 275,000 deaths every year. RHD is still a major problem in developing countries. PTMC, also known as percutaneous mitral balloon valvotomy, is the first choice of treatment for symptomatic patients with rheumatic mitral valve stenosis. PTMC using the Inoue balloon was first developed by Inoue in $1982^{5}$. As with other interventional procedures, there is a certain learning curve for mastering PTMC technique using the Inoue balloon catheter, and teaching catheter intervention techniques is a challenge. Simulationbased training has now been widely accepted as an important tool for procedural training and is seen as a significant future development for interventional cardiology training programs worldwide. The novel simulator presented in this paper does not require a power supply, it can be used with a small amount of water, and it is portable and easy to assemble within one hour. We developed this simulator for use in developing countries with limited power and water supplies.

The training programme also included observation and performance of PTMC in actual patients. During the training period, a total of 25 patients underwent PTMC (NICVD: 13 patients, NKH 12 patients) where the Inoue balloon catheter was used by Dr. Inoue himself or by the training participants under his supervision (Figure 5).

\section{Limitations}

The present study has several important limitations. First, evaluation of the simulation was done by a questionnaire, which is subjective and may have poor reproducibility. Second, most participants had already carried out PTMC using other balloon

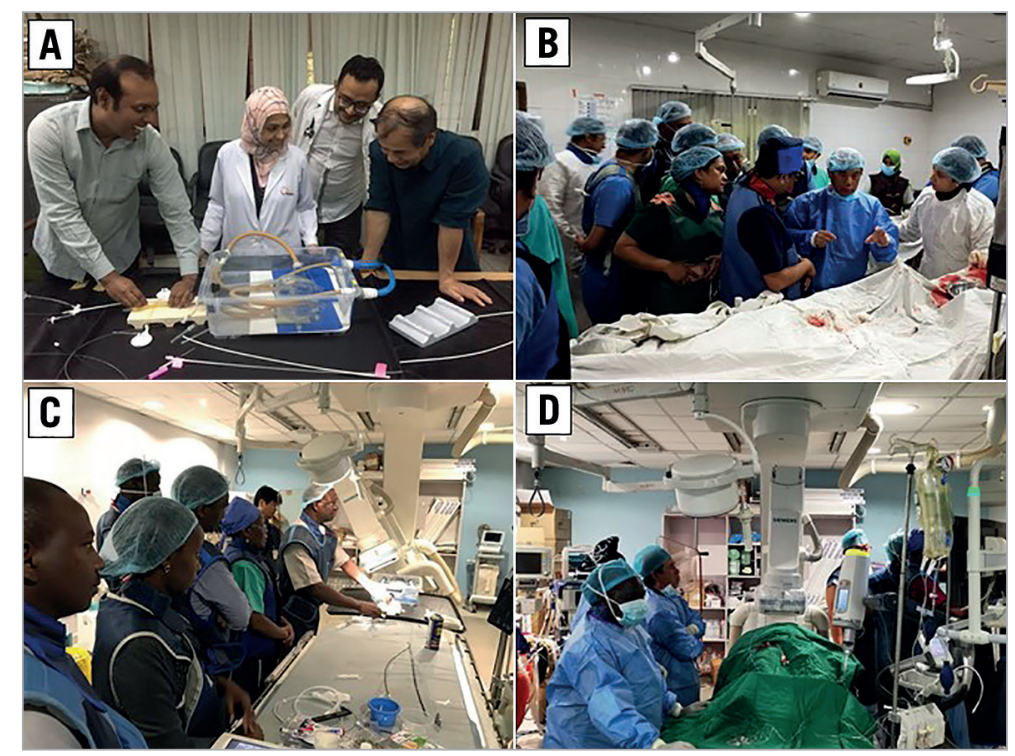

Figure 5. Simulation training and observation and education in an actual percutaneous transvenous mitral commissurotomy (PTMC). A) Simulation training in National Institute of Cardiovascular Disease (NICVD; B) Actual PTMC in NICVD; C) Simulation training in Kenyatta National Hospital (KNH); D) Actual PTMC in KNH. 
procedures, which could have influenced the study results. Third, the 3D-printed heart models used in this study were created from patients with typical mitral stenosis, but the variation in heart morphology is much greater in real patients.

\section{Conclusions}

A novel assembly catheter simulator using a 3D-printed heart model with a foot pump to create pulsatile flow for PTMC is useful for training physicians in developing countries.

\section{Impact on daily practice}

A novel portable assembly catheter simulator using a 3D-printed heart model with a foot pump to create pulsatile flow was developed. The simulator can be used with a small amount of water, and it is portable and easy to assemble. This simulator will be utilised in many developing countries for training of complex catheter procedures.

\section{Acknowledgements}

We thank Mr. Atsushi Yamazaki for his commitment and collaboration in this project. We would like to thank the NICVD and KNH staff who cooperated in this training. We also acknowledge the generous support of the Ministry of Health, Labour and Welfare in Japan, and JICA.

\section{Funding}

The training in Bangladesh was conducted as part of an international programme supported by the Ministry of Health, Labour and Welfare, Japan, and the training in Kenya was conducted as part of an international programme supported by the Japan International Cooperation Agency (JICA).

\section{Conflict of interest statement}

The authors have no conflicts of interest to declare.

\section{References}

1. Watkins DA, Johnson CO, Colquhoun SM, Karthikeyan G, Beaton A, Bukhman G, Forouzanfar MH, Longenecker CT, Mayosi BM, Mensah GA, Nascimento BR, Ribeiro ALP, Sable CA, Steer AC, Naghavi M, Mokdad AH, Murray CJL, Vos T, Carapetis JR, Roth GA. Global, Regional, and National Burden of Rheumatic Heart Disease, 1990-2015. N Engl J Med. 2017;377:713-22.

2. Marijon E, Mirabel M, Celermajer D, Jouven X. Rheumatic heart disease. Lancet. 2012;379:953-64.

3. Tefera E, Qureshi SA, Bermudez-Cañete R. Successful training of self-sufficient interventional paediatric cardiology team in a subSaharan setting: A multicentre collaborative model. Cardiol Young. 2015;25:874-8.

4. Zühlke L, Karthikeyan G, Engel ME, Rangarajan S, Mackie P, Cupido-Katya Mauff B, Islam S, Daniels R, Francis V, Ogendo S, Gitura B, Mondo C, Okello E, Lwabi P, Al-Kebsi MM, Hugo-Hamman C, Sheta SS, Haileamlak A, Daniel W, Goshu DY, Abdissa SG, Desta AG, Shasho BA, Begna DM, Elsayed A, Ibrahim AS, Musuku J, Bode-Thomas F, Yilgwan CC, Amusa GA, Ige O, Okeahialam B, Sutton C, Misra R, Abul Fadl A, Kennedy N, Damasceno A, Sani MU, Ogah OS, Elhassan TOHM, Mocumbi AO, Adeoye AM, Mntla P, Ojji D, Mucumbitsi J, Teo K, Yusuf S, Mayosi BM. Clinical Outcomes in 3343 Children and Adults with Rheumatic Heart Disease from 14 Low-and Middle-Income Countries: Two-Year Follow-Up of the Global Rheumatic Heart Disease Registry (the REMEDY Study). Circulation. 2016;134:1456-66.

5. Inoue K, Owaki T, Nakamura T, Kitamura F, Miyamoto N. Clinical application of transvenous mitral commissurotomy by a new balloon catheter. J Thorac Cardiovasc Surg. 1984;87:394-402. 